\title{
Tangier disease as a test of the reverse cholesterol transport hypothesis
}

\author{
Alan R. Tall and Nan Wang \\ Division of Molecular Medicine, Department of Medicine, Columbia University, New York, New York, USA
}

Address correspondence to: Alan R. Tall, Columbia University, Department of Medicine, Division of Molecular Medicine, New York, New York 10032, USA. Phone: (212) 305-4899; Fax: (212) 305-5052; E-mail: art1@columbia.edu.

Atherosclerotic cardiovascular disease is the major killer in industrialized societies. Treatment of this disorder via LDL lowering, particularly with statin drugs, has been partially effective but no panacea. Many patients developing coronary heart disease (CHD) do not have markedly elevated LDL but rather have low HDL levels, alone or accompanied by hypertriglyceridemia. The strong inverse relationship between HDL levels and atherosclerotic cardiovascular disease has been known for more than 25 years (1), but, surprisingly, no effective therapy specifically directed at HDL has yet been developed. This reflects uncertainty about the mechanisms underlying the protective effect of HDL, and also a lack of attainable therapeutic targets. The oldest theory to explain HDL's protective relationship is the reverse cholesterol transport (RCT) hypothesis, which holds that HDL mediates the movement of cholesterol from peripheral cells, including macrophage-derived foam cells in the arterial wall, back to the liver, where it is excreted into bile (2) (Figure 1).

The RCT theory evolved from an understanding of the role of HDL in transporting cholesterol through the bloodstream to the liver and the finding that HDL and its major apolipoprotein, apoA-I, could remove cholesterol from foam cells $(3,4)$. A number of other properties of HDL could also be involved in its protective effect. Thus, HDL can suppress expression of cytokine-induced endothelial cell adhesion molecules and block the migration of macrophages into the subendothelial space of blood vessels. HDL protects LDL from oxidation by a variety of mechanisms and may serve as an anticoagulant and antiplatelet agent (see ref. 5). To what extent the various properties of HDL influence the progression of cardiovascular disease remains uncertain.

Evidence for a direct protective effect of HDL in atherosclerosis came with the development of transgenic mice overexpressing the major HDL apoprotein, apoA-I. Transgenic mice expressing human apoA-I have increased HDL levels and are substantially protected from developing atherosclerosis, induced either by diet or genetic manipulation (5). Furthermore, serum from apoA-I transgenic rats stimulates cellular cholesterol efflux to a greater extent than normal serum (6), and apoA-I transgenic mice have increased transport of cholesterol to the liver (7); apoAI knockout mice, conversely, have decreased flux (8). Placing the apoA-I transgene in the background of the otherwise proatherogenic apoE deletion genotype yields mice that are protected from atherosclerosis, even though they show no alteration of endothelial adhesion molecule (VCAM-1, ICAM-1) expression in lesions or of oxidationassociated epitopes in plasma (9). While not conclusive, these findings are consistent with increased RCT as the protective mechanism in these animals.

\section{HDL and human atherogenesis}

Evidence relating HDL, RCT, and atherogenesis in humans has been even more difficult to obtain. Human genetic deficiency states affecting HDL levels should offer insight into this question. An appreciable fraction of subjects with low HDL due to rare apoA-I gene mutations have premature atherosclerosis, but many of these subjects do not. Mutations in the plasma cholesteryl ester transfer protein (CETP), which facilitates the return of HDL cholesterol to the liver via the triglyceride-rich lipoproteins (Figure 1), result in increased HDL levels (10). Heterozygotes with this mutation may have increased CHD risk, consistent with the RCT theory (11). However, homozygous CETP deficiency with very high HDL levels appears to be associated with decreased CHD risk (11), probably reflecting additional protective effects operating at high HDL levels.

Studies in Tangier disease (TD) have shed further light on this complex question. Homozygous TD is a rare disorder associated with extremely low levels of HDL cholesterol (less than 5\% of normal) and apoA-I (less than 1\%). The hallmark pathology is cholesteryl ester (CE) accumulation in tissue macrophages, leading to accumulation of foam cells in various organs, such as the liver and spleen. Importantly, fibroblasts from Tangier subjects show a dramatic reduction in cholesterol and phospholipid efflux to apoA-I, indicating a defect in the initial step of RCT as the key cellular defect (12). Moreover, a systematic survey of TD patients suggests that homozygotes have an approximately four- to sixfold increased risk of atherosclerotic cardiovascular disease, compared with age-matched controls (13). While not as striking as the increased risk seen in familial hypercholesterolemia, Tangier homozygotes also have reduced LDL cholesterol ( $40 \%$ of normal), which may partly offset the decrease in RCT. Obligate heterozygotes for the TD mutation have decreased HDL cholesterol (about $50 \%$ of normal), normal LDL cholesterol, and an apparent increase in risk for atherosclerosis (13).

\section{The ABCs of RCT}

Recently, TD was shown to be caused by mutation in an ATP binding cassette transporter, ABCA1 (or ABC1) (14-16). This finding immediately provided a potential explanation for the cellular defect, since ABCA1 stimulates cholesterol and phospholipid efflux to apoAI and may act as a cholesterol/phospholipid flippase at the plasma membrane (17-19).

In this issue of the JCI, Clee et al. (20) provide important further evidence strengthening the link between defec- 


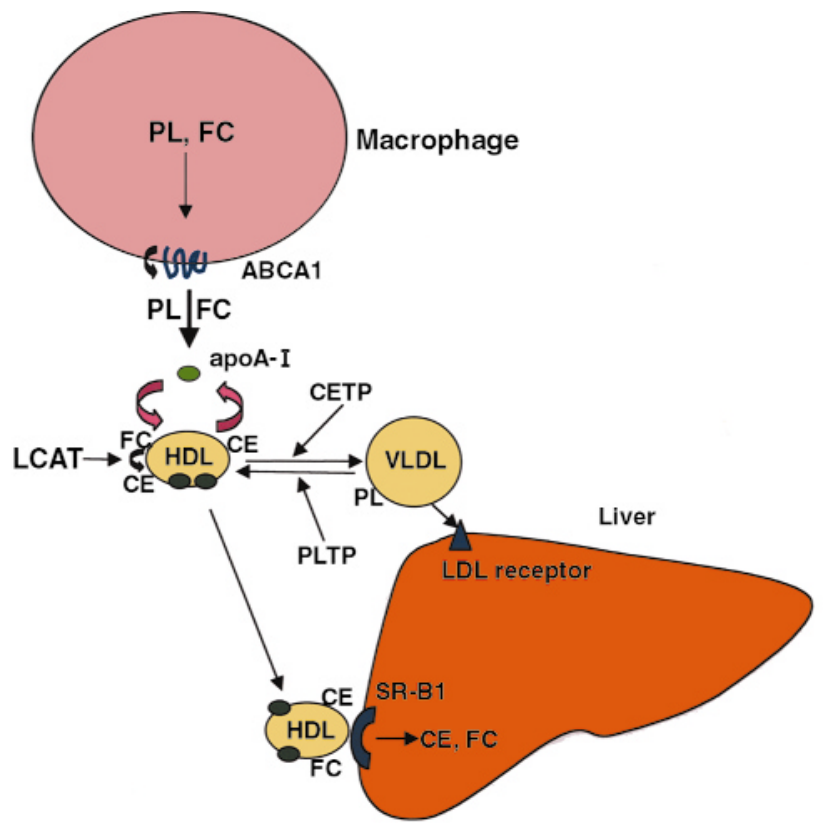

Figure 1

HDL-mediated RCT. ABCA1 is involved in transfer of free cholesterol $(F C)$ and phospholipids (PL) from macrophages to apoA-I. Lecithin cholesterol acyltransferase (LCAT) converts FC to CE. CETP and PLTP modify HDL by transferring CE and PL between HDL and triglyceride-rich lipoproteins. HDL delivers cholesterol to liver via SR-BI-mediated selective uptake of the lipids.

tive cellular cholesterol efflux, low HDL, and increased atherosclerosis in TD. Studying 13 different $A B C A 1$ mutations in 11 different families, these authors show a more than threefold excess of coronary artery disease (CAD) in affected family members, compared with unaffected members. Moreover, CAD appears at an earlier age in the subjects with $A B C A 1$ mutations. This kind of study has as a strength that unaffected siblings tend to share genetic and environmental risk factors, making them a reasonable control group, although the number of subjects remains small, and the approach potentially suffers from the problem of selection bias. In this case, two TD probands presented with $\mathrm{CAD}$, raising the concern that the study overestimates the effects of the mutation. However, $A B C A 1$ mutations are fairly common, raising the hope that future population studies of mutations with defined functional effects may eventually provide more conclusive evidence concerning the relationship of ABCA1 mutations and atherosclerosis. Further insights will be gained from studies in $A B C A 1$ knockout mice, which have low HDL and increased tissue foam cells (21); crosses of these mice with atherosclerosis-susceptible strains are likely to be informative.
A key aspect of this study (20) is the demonstration of a relationship between the defect in cellular cholesterol efflux and HDL levels in subjects with heterozygous $A B C A 1$ mutations. Heterozygotes were found to have wide variation in HDL levels, with the low HDL phenotype becoming more evident at later age. Variations in cholesterol efflux in a fibroblast assay correlated well with HDL levels. Together with the results of an earlier study (22), these findings indicate that variation in ABCA1 function leads to different levels of cellular cholesterol efflux and correlates with HDL levels and HDL size. HDL levels in heterozygotes are comparable to those seen in epidemiological studies, providing plausibility to the theory that differences in efficiency of RCT underlie the relationship between HDL and cardiovascular disease in at least some part of the general population. However, it is likely that low HDL in the general population represents a diversity of different disorders, in some cases reflecting defective RCT, and in other cases reflecting increased remnant levels or defective apoA-I synthesis. The development of molecular markers for the underlying defects should allow more suitable classification of low HDL disorders in the future.
It is perhaps surprising that differences in ABCA1 expression have such a large effect on plasma HDL levels. ABCA1 stimulates cholesterol and phospholipid efflux to free apoA-I, but has only a minor effect on efflux to HDL (17). ABCA1 binds and can be crosslinked to free apoA-I but not HDL. These findings are consistent with earlier studies suggesting that a minor, pre- $\beta$ HDL fraction (which includes free or minimally lipidated apoA-I) plays a unique role in stimulating the initial phase of cellular cholesterol efflux (23). Strikingly, ABCA1 appears to be saturated at very low concentrations of free apoA-I (less than $15 \mu \mathrm{g} / \mathrm{ml}$ ) (17); this compares with a plasma concentration of apoA-I of about $1 \mathrm{mg} / \mathrm{ml}$, of which about $60-120 \mu \mathrm{g} / \mathrm{ml}$ might be in the form of pre- $\beta$ HDL or free apoAI. This plasma concentration would be more than adequate to saturate cellular ABCA1, but the concentration of free apoA-I in atherosclerotic lesions, where foam cells reside, is unknown. Nevertheless, a recent report indicates that the concentration of pre- $\beta$ HDL in human peripheral lymph is about 15 $\mu \mathrm{g} / \mathrm{ml}$ (24). Therefore, it seems likely that a very small pool of free apolipoprotein (apoA-I or apoA-IV) acts as the substrate for ABCA1. This free apoA-I may be secreted from the liver or small intestine or may be regenerated in the circulation as a result of remodeling of HDL by CETP, followed by the action of hepatic lipase. Both the concentration of free apoA-I in tissues and the level of ABCA1 expression in cells may be critical for the initial lipidation of apoA-I. Low levels of ABCA1 expression result in reduced lipidation and hypercatabolism of apoA-I. A second stage of maturation of HDL involves the actions of lecithin cholesterol acyltransferase (LCAT) and phospholipid transfer protein (PLTP), leading to the formation of mature plasma HDL (25). Large, apoE-rich HDLs appear to be the optimal substrates for scavenger receptor BI (SR-BI), which plays a major role in extracting CEs from particles in the liver $(26,27)$.

A striking feature of ABCA1 gene expression is its marked upregulation in foam cells (28), which was recently shown to be mediated by binding of the oxysterol-activated nuclear hormone receptor LXR, in combination with RXR, to a direct repeat four element in the proximal promoter $A B C A 1$ 
(29). Thus, LXRs may serve to coordinate different steps in RCT, including ABCA1, CETP, and cyp7a. A role for ABCA1 in the regulation of cholesterol absorption has also recently been suggested $(21,30)$. Additionally, ABCA1 expression is downregulated by IFN- $\gamma$, hinting at a link between the inflammatory component of atherosclerotic lesions and a defect in RCT (31).

\section{Prospects}

We are at the beginning of a new era in HDL research. The RCT hypothesis has received a big boost from the elucidation of the Tangier mutation and from the studies reported in this issue of the JCI (20). Plasma HDL levels now appear to be important as a marker of the efficiency of ABCA1-mediated cholesterol removal from cells, a model that is related to, but distinct from, the idea that high HDL is beneficial because the bulk HDL extracts cholesterol directly from cells. Although there are probably several different antiatherogenic properties of HDL, these considerations suggest that therapeutic interventions to raise HDL should be evaluated with regard to mechanism and ultimately will need confirmation of efficacy using clinical endpoints. Importantly, we now have a molecular framework for understanding the RCT pathway, which will lead to new opportunities for therapeutic intervention and many interesting questions concerning the cellular mechanisms for regulating cholesterol efflux.

1. Rhoads, G.G., Gulbrandsen, C.L., and Kagan, A. 1976. Serum lipoproteins and coronary heart disease in a population study of Hawaii Japanese men. N. Engl. J. Med. 294:293-298.

2. Glomset, J.A. 1980. High-density lipoproteins in human health and disease. Adv. Intern. Med. 25:91-116.
3. Ho, Y.K., Brown, M.S., and Goldstein, J.L. 1980 Hydrolysis and excretion of cytoplasmic cholesteryl esters by macrophages: stimulation by high density lipoprotein and other agents. J. Lipid Res. 21:391-398.

4. Hara, H., and Yokoyama, S. 1991. Interaction of free apolipoproteins with macrophages. Formation of high density lipoprotein-like lipoproteins and reduction of cellular cholesterol. J. Biol. Chem. 266:3080-3086.

5. Lusis, A.J. 2000. Atherosclerosis. Nature. 407:233-241.

6. Fournier, N., et al. 1996. Role of HDL phospholipid in efflux of cell cholesterol to whole serum: studies with human apoA-I transgenic rats. J. Lipid Res. 37:1704-1711.

7. Khoo, J.C., Pittman, R.C., and Rubin, E.M. 1995. Selective uptake of HDL cholesteryl esters is active in transgenic mice expressing human apolipoprotein A-I. J. Lipid Res. 36:593-600.

8. Plump, A.S., et al. 1997. ApoA-I knockout mice: characterization of HDL metabolism in homozygotes and identification of a post-RNA mechanism of apoA-I up-regulation in heterozygotes. $J$. Lipid Res. 38:1033-1047.

9. Dansky, H.M., et al. 1999. Apo A-I inhibits foam cell formation in Apo E-deficient mice after monocyte adherence to endothelium. J. Clin. Invest. 104:31-39.

10. Inazu, A., et al. 1990. Increased high-density lipoprotein levels caused by a common cholesteryl-ester transfer protein gene mutation. $N$. Engl.J. Med. 323:1234-1238.

11. Zhong, S., et al. 1996. Increased coronary heart disease in Japanese-American men with mutation in the cholesteryl ester transfer protein gene despite increased HDL levels. J. Clin. Invest. 97:2917-2923.

12. Francis, G.A., Knopp, R.H., and Oram, J.F. 1995. Defective removal of cellular cholesterol and phospholipids by apolipoprotein A-I in Tangier Disease. J. Clin. Invest. 96:78-87.

13. Schaefer, E.J., Zech, L.A., Schwartz, D.E., and Brewer, H.B., Jr. 1980. Coronary heart disease prevalence and other clinical features in familial high-density lipoprotein deficiency (Tangier disease). Ann. Intern. Med. 93:261-266.

14. Brooks-Wilson, A., et al. 1999. Mutations in ABC1 in Tangier disease and familial high-density lipoprotein deficiency. Nat. Genet. 22:336-345.

15. Bodzioch, M., et al. 1999. The gene encoding ATP-binding cassette transporter 1 is mutated in Tangier disease. Nat. Genet. 22:347-351.

16. Rust, S., et al. 1999. Tangier disease is caused by mutations in the gene encoding ATP-binding cassette transporter 1. Nat. Genet. 22:352-355.

17. Wang, N., Silver, D.L., Costet, P., and Tall, A.R. 2000. Specific binding of apoA-I, enhanced cholesterol efflux and altered plasma membrane morphology in cells expressing ABC1. J. Biol. Chem. In press.
18. Bortnick, A.E., et al. 2000. The correlation of ATP-binding cassette $1 \mathrm{mRNA}$ levels with cholesterol efflux from various cell lines. J. Biol. Chem. 275:28634-28640.

19. Oram, J.F., Lawn, R.M., Garver, M.R., and Wade, D.P. 2000 . ABCA1 is the cAMP-inducible receptor that mediates cholesterol secretion from macrophages. J. Biol. Chem. In press.

20. Clee, S.M., et al. 2000. Age and residual cholesterol efflux affect HDL cholesterol levels and coronary artery disease in $A B C A 1$ heterozygotes. J. Clin. Invest. 106:1263-1270.

21. McNeish, J., et al. 2000. High density lipoprotein deficiency and foam cell accumulation in mice with targeted disruption of ATP-binding cassette transporter-1. Proc. Natl. Acad. Sci. USA. 97:4245-4250.

22. Brousseau, M.E., et al. 2000. Cellular cholesterol efflux in heterozygotes for tangier disease is markedly reduced and correlates with high density lipoprotein cholesterol concentration and particle size. J. Lipid Res. 41:1125-1135.

23. Castro, G.R., and Fielding, C.J. 1988. Early incorporation of cell-derived cholesterol into pre-betamigrating high-density lipoprotein. Biochemistry. 27:25-29.

24. Nanjee, M.N., Cooke, C.J., Olszewski, W.L., and Miller, N.E. 2000. Concentrations of electrophoretic and size subclasses of apolipoprotein A-I-containing particles in human peripheral lymph. Arterioscler. Thromb. Vasc. Biol. 20:2148-2155.

25. Jiang, X.C., et al. 1999. Targeted mutation of plasma phospholipid transfer protein gene markedly reduces high-density lipoprotein levels. J. Clin. Invest. 103:907-914.

26. Arai, T., et al. 1999. Decreased selective uptake of high density lipoprotein cholesteryl esters in apolipoprotein E knock-out mice. Proc. Natl. Acad. Sci. USA. 96:12050-12055.

27. Rigotti, A., et al. 1997. A targeted mutation in the murine gene encoding the high density lipoprotein (HDL) receptor scavenger receptor class B type I reveals its key role in HDL metabolism. Proc. Natl. Acad. Sci. USA. 94:12610-12615.

28. Langmann, T., et al. 1999. Molecular cloning of the human ATP-binding cassette transporter 1 (hABC1): evidence for sterol-dependent regulation in macrophages. Biochem. Biophys. Res. Commun. 257:29-33.

29. Costet, P., Luo, Y., Wang, N., and Tall, A.R. 2000 Sterol-dependent transactivation of the ABC1 promoter by the liver X Receptor/Retinoid X receptor. J. Biol. Chem. 275:28240-28245.

30. Repa, J.J., et al. 2000. Regulation of absorption and $\mathrm{ABC} 1$-mediated efflux of cholesterol by RXR heterodimers. Science. 289:1524-1529.

31. Panousis, C.G., and Zuckerman, S.H. 2000. Interferon-gamma induces downregulation of Tangier disease gene (ATP-binding-cassette transporter 1) in macrophage-derived foam cells. Arterioscler. Thromb. Vasc. Biol. 20:1565-1571. 